\title{
Automatic identification of bird females using egg phenotype
}

2 Michal Šulc ${ }^{1}$, Anna E. Hughes ${ }^{2}$, Jolyon Troscianko³ ${ }^{3}$ Gabriela Štětková ${ }^{1,4}$, Petr Procházka ${ }^{1}$, Milica

3 Požgayová ${ }^{1}$, Lubomír Piálek ${ }^{1,5}$, Radka Piálková $^{1,5}$, Vojtěch Brlík ${ }^{1,6}$ and Marcel Honza ${ }^{1}$

4

$5{ }^{1}$ Czech Academy of Sciences, Institute of Vertebrate Biology, Brno, Czech Republic

62 Department of Psychology, University of Essex, Colchester, U.K.

$7 \quad{ }^{3}$ Centre for Life and Environmental Sciences, University of Exeter, Penryn, U.K.

$8{ }^{4}$ Department of Botany and Zoology, Faculty of Sciences, Masaryk University, Brno, Czech Republic

$9{ }^{5}$ Faculty of Natural Sciences, University of South Bohemia, České Budějovice, Czech Republic

$10{ }^{6}$ Department of Ecology, Faculty of Science, Charles University, Prague, Czech Republic

11 Corresponding author: sulc@ivb.cz

\section{Abstract}

1. Identification of individuals greatly contributes to understanding animal ecology and evolution, and in many cases can only be achieved using expensive and invasive techniques. Advances in computing technology offer alternative cost-effective techniques which are less invasive and can discriminate between individuals based on visual and/or acoustic cues. Here, we employ human assessment and an automatic analytical approach to predict the identity of common cuckoo (Cuculus canorus) females based on the appearance of their eggs. The cuckoo's secretive brood parasitic strategy makes studying its life history very challenging. Eggs were analysed using calibrated digital photography for quantifying spotting pattern, size and shape, and spectrometry for measuring colour. Cuckoo females were identified from genetic sampling of their nestlings, allowing us to determine the accuracy of human and automatic female assignment. Finally, we used a novel 'same-different' approach that uses both genetic and phenotypic information to assign eggs that were not genetically analysed. 
2. Our results supported the 'constant egg-type hypothesis', showing that individual cuckoo females lay eggs with a relatively constant appearance and that eggs laid by different females differ more than eggs laid by the same female. The accuracy of unsupervised hierarchical clustering was comparable to assessments of experienced human observers. Supervised random forest analysis showed better results, with higher cluster accuracy. Same-different analysis was able to assign 22 of 87 unidentified cuckoo eggs to seven already known females.

3. Our study showed that egg appearance on its own is not sufficient for identification of individual cuckoo females. We therefore advocate genetic analysis to be used for this purpose. However, supervised analytical methods reliably assigned a relatively high number of eggs without genetic data to their mothers which can be used in conjunction with genetic testing as a cost-effective method for increasing sample sizes for eggs where genetic samples could not be obtained.

43 Identification of individuals is important in zoological research, particularly when investigating

44 variation among or within individuals in a population. Traditionally, capture-mark-recapture

45 techniques have been used to monitor individuals during their lifetime (Lindberg, 2012; Jung,

46 Boonstra, \& Krebs, 2020). This method has been improved by employing more sophisticated

47 methods such as attaching GPS (global positioning system) and radio transmitters or RFID (radio

48 frequency identification) tags (Krause et al., 2013) that allow researchers to investigate the spatial

49 activity of animals in more detail. However, these methods still require capturing and tagging that

50 is usually time-consuming, expensive (depending on the method used), and may reduce animal

51 welfare (Weinstein, 2018). Therefore, cost-effective indirect approaches have been developed to 52 identify and monitor individuals within the same species. 
54 These indirect approaches rely on the fact that individuals differ from each other visually or 55 acoustically and this variation may be used for their identification. Indeed, it has been shown that e.g. face (Deb et al., 2018; Hansen et al., 2018; Hou et al., 2020) and body pattern data (Hiby et al., 2009; Bolger, Morrison, Vance, Lee, \& Farid, 2012; Crall, Stewart, Berger-Wolf, Rubenstein, \& Sundaresan, 2013; Ferreira et al., 2020) captured from photographs may allow discrimination of individuals. Similarly, sounds produced by animals (especially bird song) also seem to serve as a good individual fingerprint (Blumstein et al., 2011; Petrusková, Pišvejcová, Kinštová, Brinke,

61 \& Petrusek, 2016; Ptacek, Machlica, Linhart, Jaska, \& Muller, 2016). Recently, applying modern computer technology and artificial intelligence techniques (such as convolutional neural networks) that automate the analysis of various types of data from different sources such as pictures or audio recordings has made these methods reliable and applicable for various animal taxa (Hansen et al., 2018; Christin et al., 2019; Ferreira et al., 2020; Hou et al., 2020).

However, for many species, identification of all individuals in a population is still not straightforward e.g. due to their secretive behaviour or due to the fact that it is difficult to catch them. Here, we focus on one group of animals that are especially challenging to study - avian brood parasites. There are more than a hundred obligate brood parasites that never build their own nests and instead lay their eggs into nests of other species (Davies, 2010). Even more species belong to conspecific brood parasites that only occasionally lay eggs into nests of the unrelated conspecifics (Bruce E. Lyon \& Eadie, 2008). Brood parasites and their hosts have been

74 the focus of considerable research into co-evolutionary arms races (Soler, 2017). But since brood 75 parasites only lay eggs and then usually do not return to host nests (but see Šulc et al. 2020), 76 and because egg laying is (especially in obligate brood parasites) very quick (McMaster, Neudorf,

77 Sealy, \& Pitcher, 2004; Gloag, Fiorini, Reboreda, \& Kacelnik, 2013; Jelínek, Šulc, Štetková, \& 78 Honza, 2020), direct observation of parasitism in nature is difficult and identification of parasitic 
79 females is problematic. As a consequence, many important aspects of females' life history

80 strategy are still poorly understood; in obligate brood parasites, we e.g. still know little about

81 spatio-temporal distribution of their egg laying, consistency in host selection and the total number

82 of eggs they lay during a breeding season. Conspecific brood parasitism is even less understood

83 and we do not even know why some females adopt this strategy (Bruce E. Lyon \& Eadie, 2008).

85 The idea of identifying bird females according to the appearance of the eggs they laid depends

86 on the presumption that within-clutch variation in egg appearance is lower than between-clutch

87 variation which has been confirmed for several species including brood parasites (Øien, Moksnes,

88 \& Røskaft, 1995; McRae \& Burke, 1996; Paillisson, Latraube, Marion, \& Bretagnolle, 2008; Höltje,

89 Mewes, Haase, \& Ornés, 2016). This approach has therefore been applied (although with

90 caveats) for the identification of parasitic eggs in some conspecific brood parasite species

91 (Gibbons, 1986; Møller, 1987; Jackson, 1992; Petersen, 1992; Bruce E. Lyon, 1993; McRae \&

92 Burke, 1996; B. E Lyon, 2003). However, some studies that estimated accuracy of parasitic egg

93 identification showed ambiguous results for some species (Ådahl, Lindström, Ruxton, Arnold, \&

94 Begg, 2004; Pöysä, Lindblom, Rutila, \& Sorjonen, 2009; Eadie, Smith, Zadworny, Kühnlein, \&

95 Cheng, 2010; Lemons, Sedinger, \& Randle, 2011; Petrželková, Pöysä, Klvaňa, Albrecht, \& Hořák,

96 2017) and for others this method did not work at all (Brown \& Sherman, 1989; Cariello, Lima,

97 Schwabl, \& Macedo, 2004; Grønstøl, Blomqvist, \& Wagner, 2006; Griffith, Barr, Sheldon, Rowe,

98 \& Burke, 2009; Roy, Parker, \& Gates, 2009). One of the reasons why many studies found low

99 accuracy of identification might be that closely related females lay indistinctive eggs. Several

100 studies showed that egg appearance, namely egg color (Wei, Bitgood, \& Dentine, 1992; Collias,

101 1993; Morales et al., 2010), spotting pattern (Gosler, Barnett, \& James Reynolds, 2000) and egg

102 size (Christians, 2002) are highly heritable traits which might complicate such analyses especially

103 in inbred populations. Another explanation might be that previous studies did not use the full 
104 potential of egg variability (e.g. none of the presented studies measured egg colour in the

105 ultraviolet range of spectrum).

107 Identification of parasitic females using egg appearance has also been attempted in the common

108 cuckoo (hereafter cuckoo), but was unsuccessful (Moksnes et al., 2008). However, the study

109 assessed cuckoo eggs from a human perspective, with people sorting the eggs based on

110 photographs. To date, there have been no attempts to use more objective quantification methods

111 for egg classification in the cuckoo. These objective methods, such as spectrophotometry for

112 measuring colours (including the ultraviolet part of the spectrum), and image analysis of

113 photographs for quantifying spotting pattern, size and shape of eggs are now available, and may

114 allow more accurate classification that can be carried out in an automated manner.

116 In this study, we employ a novel automatic analytical approach to analyse phenotypic features of

117 cuckoo eggs such as dimensions (size, shape), colour and spotting pattern to predict maternal

118 identity. If successful, this low-cost and minimally invasive female identification method would

119 greatly facilitate studies into a range of key questions regarding this secretive brood parasitic

120 species. We also performed human assessment based on sorting of photos with cuckoo eggs to

121 compare the reliability of both methods with the true identity acquired from molecular analyses.

122 Moreover, we believe this automatic technique might be also used in other brood parasitic

123 systems or in species where females are difficult to catch (see e.g. Höltje et al. 2016). Finally, it

124 has been suggested that similarly looking eggs laid by different cuckoo females may belong to

125 closely related females, e.g. mother and daughter (Moksnes et al., 2008). Therefore, we will for

126 the first time investigate the relationship between the genetic distance of individual cuckoo

127 females and the phenotypic distance of their eggs. 


\section{Material and Methods}

\section{Study system and data collection}

132 All data were collected in the fishpond area between Mutěnice $\left(48^{\circ} 54^{\prime} \mathrm{N}, 17^{\circ} 02^{\prime} \mathrm{E}\right)$ and Hodonín $133\left(48^{\circ} 51^{\prime} \mathrm{N}, 17^{\circ} 07^{\prime} \mathrm{E}\right)$ in South Moravia, Czech Republic from May to July 2017 . We systematically

134 searched the littoral vegetation for the great reed warbler (Acrocephalus arundinaceus) and

135 Eurasian reed warbler (Acrocephalus scirpaceus) nests. Most great reed warbler (hereafter GRW)

136 nests were found during the building stage when mapping male territories and mating status

137 (Bensch, 1996). The rest of the GRW and all Eurasian reed warbler (hereafter RW) nests were

138 found in different stages of breeding by systematic searching. If possible, all GRW nests were

139 checked every day from the nest building stage until clutch completion and approximately every

140 third day during incubation. All RW nests were checked approximately every second day during

141 laying stage and extensively during incubation. GRWs experienced $92 \%$ (59 out of 64 nests) and

142 RWs $20 \%$ (91 out of 456 nests) cuckoo parasitism rate. Multiple parasitism was also common;

14337 of 59 and two of 91 parasitized GRW and RW nests, respectively, were parasitized by more

144 than one cuckoo egg.

146 When a cuckoo egg was found in a host nest, we immediately measured its colour and took a 147 photo (see below) to avoid colour change during the incubation period (Hanley et al., 2016). In 148 the cases of multiply parasitized nests, we removed the newly laid cuckoo egg(s), transferred 149 them to an incubator (HEKA-Kongo; HEKA-Brutgeräte, Rietberg, Germany) and incubated them 150 artificially to prevent sample losses caused by the cuckoo nestlings (Honza, Vošlajerová, \& 151 Moskát, 2007). The removed cuckoo eggs were either incubated until hatching and chicks placed 152 into non-parasitized host nests (for other experiments) or we froze the eggs before hatching for 153 the future genetic analysis (see Genotyping and kinship analysis section). We took a blood 154 sample from all 10-days old cuckoo nestlings from their ulnar or medial tarsometatarsal vein 155 (approx. $25 \mu \mathrm{l}$ ). Finally, we mist-netted 29 and 16 adult cuckoo males and females, respectively, 
and collected their blood samples from the ulnar vein (approx. $25 \mu \mathrm{l}$ ). All blood samples were

157 stored in $96 \%$ ethanol until later genetic analyses.

Altogether we found 203 cuckoo eggs (121 and 82 in the GRW and RW nests, respectively). We photographed and measured the colour of 192 of them. Among these photographed cuckoo eggs, genetic samples were collected from 105 nestlings or embryos.

Measurements of egg appearance

164 To obtain background colour we measured reflectance using JAZ Spectrometer (Ocean Optics,

165 Dunedin, FL, U.S.A.) in the range $300-700 \mathrm{~nm}$, as that is the wavelengths range birds can perceive (Cuthill 2006). We took nine measurements (each covering approximately $1 \mathrm{~mm}^{2}$ ) at three different parts of the egg (sharp pole, middle part and blunt pole). Since we focused on background colour, we avoided measuring dark spots (Šulc et al. 2016). For each egg, we used the measurement with the highest reflectance that best corresponded to the colour of the background (Šulc et al. 2019).

Spotting pattern was calculated from digital images taken by a Canon EOS 700D with prime

173 Canon EF $40 \mathrm{~mm}$ lens. All photos were taken under standardized diffuse sunlight conditions

174 (using a photography light tent), at the same angle and from the same distance and were referred 175 to a grey standard (X-Rite Colour Checker Grey Scale Chart) with known reflectance. Image 176 calibration, pattern analysis, analysis of shape and measurements of size were performed in 177 ImageJ software (Schneider et al. 2012) using the Multispectral Image Calibration and Analysis 178 (MICA) Toolbox (van den Berg et al. 2020). A scale bar was included in each photo, meaning that 179 all images were equally rescaled to the scale of the smallest image (30 pixels $/ \mathrm{mm}$ ), and egg 180 dimensions were obtained from the photos. For pattern investigation we applied a granularity 181 analysis approach (Troscianko and Stevens 2015) that creates a bandpass 'energy' spectrum 
across a range of spatial frequencies, and then the pattern energy at each frequency band was measured as the standard deviation of the filtered image (for details see Šulc et al. 2019 and van den Berg et al. 2020). Since pattern energy does not discriminate the direction of the pattern (it cannot distinguish between dark spots on light background and light spots on dark background), we also calculated the 'skew' of the pattern, which quantifies the asymmetry of the pattern

187 luminance distribution. A negative value of skew implies there are more spots than background 188 colour, while a positive skew implies there is more background colour than spots. Skew was 189 measured independently at each granularity band. All colour measurements and photos were taken by a single person (M.Š.) to ensure high consistency of the data.

\section{Genotyping and kinship analysis}

193 DNA was isolated from the blood of adults and nestlings or tissues of embryos. (65 nestlings, 41 embryos, 29 adult males and 16 adult females). We estimated kinship relationships from nuclear SNPs and mitochondrial DNA haplotypes enabling us to exclude highly implausible maternal (or maternal-sibling) relationships in the inferred genealogy. As an additional criterion we also used the laying date of cuckoo eggs (data from daily checks) because it is known that a cuckoo female cannot lay her eggs more often that every second day (Chance, 1922; Payne, 1973; Wyllie, 1981).

To acquire the SNP dataset, we genotyped all samples with the ddRAD (double digest restrictionsite associated DNA) technique (Petersen, 1992) following the protocol of (Piálek et al., 2019).

202 Two prepared libraries were sequenced on an Illumina HiSeq4000 system (2 lanes, 150 cycles $203 \mathrm{P} / \mathrm{E})$ in the EMBL Genomic Core Facility, Heidelberg, Germany. The obtained RAD-tags were 204 processed in Stacks v2.4 (Catchen et al. 2011, Rochette et al. 2019) and mapped on the Cuculus 205 canorus genome GCA000709325.1 (https://www.ncbi.nlm.nih.gov) with Bowtie2 assembler 206 v2.2.4 (Langmead \& Salzberg, 2012). Only loci with 95\% or higher presence of individuals were 207 scored and further filtered based on Hardy-Weinberg equilibrium, linkage disequilibrium and 
208 minimum minor allele frequency (0.4) in PLINK v1.9 (Purcell et al., 2007) which resulted in a 209 dataset with 1620 markers. Kinship relationships were estimated using Colony (Jones \& Wang, 2102010 ) based on $>1000$ nuclear SNPs.

212 For the mitochondrial haplotype analysis, we sequenced a 411-bp portion of the left-hand

213 hypervariable control region (Gibbs et al., 2000; Fossøy et al., 2011, 2012). Mitochondrial 214 sequence data were assembled and manually checked in Geneious v10.2.6 (Kearse et al., 2012) 215 and haplotypes were estimated based on a distance matrix with up to 1\% tolerance (approx. 4 216 mutations) for genotyping errors.

218 Kinship analysis assigned the offspring $(n=105)$ to 30 clusters containing $1-10$ eggs each (Fig.

$2191-5$ in Supplementary material). Among these 30 clusters, nine corresponded to females that 220 were caught and genotyped as described above. Thus, we were able to calculate genetic 221 distances among these females.

223 For subsequent analysis dealing with egg phenotype (human and automatic assessment, see 224 below), we removed females to which only one egg has been assigned $(n=10)$, meaning that we 225 used a final dataset of 95 eggs laid by 20 females.

\section{Human assessment}

228 We printed 95 photographs of cuckoo eggs that were standardized in their colour and size using 229 the MICA Toolbox (van den Berg et al. 2020; Fig.1-7 in supplementary material). We then asked 230 twelve people to sort these photographs and create groups of pictures representing individual 231 females according to similarity in egg appearance. Firstly, we asked them to sort these pictures 232 into an unknown number of groups and, secondly, we asked them to sort these pictures into 20 233 groups corresponding to the real number of females identified by genetic assessment of identity. 
234 For the assessments, we asked 1) five people with no experience with egg appearance from wild

235 animals, 2) three students of avian ecology that had experience with egg appearance from wild

236 birds but had never seen cuckoo eggs and 3) four people (mostly co-authors of this manuscript)

237 that had years of experience with cuckoo eggs. All participants received no other information

238 about the eggs. Cluster similarity in egg classification was assessed using the adjusted Rand

239 index, which provides a corrected-for-chance measure of the similarity between two data

240 clusterings, implemented using the 'cluster_similarity' function from the R package clustereval

241 (Ramey, 2012).

243 Automatic assessment

244 We developed an automatic method based on the similarities/differences of cuckoo egg

245 phenotypes. In the first step, we collected colour, pattern and dimension data from calibrated

246 photographs and spectrophotometry data (for details, see Šulc et al., 2019) for all cuckoo eggs.

247 Initially, we conducted Principal component analysis (PCA) on different aspects of the egg

248 photographs, in order to avoid the use of correlated variables in the models.

250 Spectral data: a spectrophotometer was used to assess the background colour of cuckoo eggs

251 (for details, see Measurements of egg appearance). PCA was carried out using binned, scaled 252 spectral data created in the R package pavo (Maia, Gruson, Endler, \& White, 2019), and two 253 spectral PCA components were used in the final dataset (based on scree plot inspection). We 254 also used two other spectral measures extracted from pavo: the mean brightness (B2; mean 255 relative reflectance over the entire spectral range) (Hill \& Mcgraw, 2006) and the position of the 256 ultraviolet (UV) peak (defined as a wavelength within the range of 300-360nm where reflectance 257 reached the highest point). 
Shape data: the variables entered into the PCA were length, maximum width, volume, ellipse deviation and surface area (Troscianko, 2014). Three shape PCA components were selected for

261 inclusion into the final dataset based on scree plot inspection.

Pattern data: the variables entered into the PCA were 12 pattern energies measured at a range

264 of scales (from 1 to 0.0221 in steps of 1/square root of 2) across the whole egg (Troscianko \&

265 Stevens, 2015), and 12 pattern energy skew values measured at the same range of scales across

266 the whole egg. We also included a measure of total pattern energy across the whole egg. Finally,

267 we divided up each egg into three segments and measured the total pattern energy in each

268 segment as well as the standard deviation between segments, to get a measure of how variable

269 the patterning was across the egg. Three pattern PCA components were selected for inclusion

270 into the final dataset based on scree plot inspection.

271

272 Luminance data: we analysed luminance from photographs, including both the spots and

273 background areas of the eggs. We divided the egg up into three segments and took the average

274 luminance and the standard deviation of luminance across each segment, as well as the standard

275 deviation of luminance across all three segments. One luminance PCA component was selected

276 for inclusion into the final dataset based on scree plot inspection.

278 In total, the final dataset contained 11 egg phenotypic traits that were used for clustering analysis.

280 Within- and between-female variability in egg appearance

281 To create a metric of within-female variance, we calculated the standard deviation for each 282 phenotypic trait within one female, and then took a mean value across all traits, giving an average 283 variability value for each female. 
284 To create a metric of between-female variance, we calculated the average value of each

285 phenotypic trait for each female (i.e. created an "average" egg) and then calculated the standard

286 deviation for each phenotypic trait across all females. We then averaged these standard

287 deviations to create a measure of between-female variability across all traits. All trait values were

288 scaled to ensure comparability across different traits.

289 To test whether within-female variance is lower than between-female variance, we conducted a 290 one-sample t-test where the within-female variance metric $(n=20)$ is compared with the test value 291 (the between-female variance value).

\section{Unsupervised learning}

294 Firstly, we carried out hierarchical clustering to attempt to cluster the eggs via visual similarity 295 without any training or further information (e.g. number of females present). All variables were 296 scaled for this analysis. To assess the accuracy of this method, we cut the tree by specifying the 297 real number of groups (20) and assessed the cluster similarity between the predictions of the 298 hierarchical model compared to the real data using the adjusted Rand index, as before.

Supervised learning

301 Female clustering: We used a random forest model with a 'leave-one-out' cross-validation 302 approach (Stone, 1974). For each egg in the dataset, the model was trained using a dataset 303 consisting of all other eggs, and then tested using the focal egg. The model attempted to classify 304 each egg to a given female, and the accuracy of the model was assessed using the classification 305 accuracy value, and through cluster similarity values, as before (taking the average of 1000 runs, 306 as random forest modelling is a stochastic process). We also fitted a random forest model to the 307 full dataset to allow us to assess the importance of the different variables included in the model 308 (using the mean decrease in accuracy). 
Same/different analysis: We used an approach where a random forest model was trained to label

311 pairs of eggs as 'same' or 'different'. The training set used 3000 'same' rows, where the two eggs

312 were from the same female (but are not identical to each other) and 3000 'different' rows, where

313 the two eggs were from different females.

315 To test our models, we tested each egg in the labelled dataset on all eggs sequentially, including

316 itself. We first tested whether the model recognised the identical eggs as being the same. We

317 then tested whether each egg was only paired with other eggs from the same female i.e. whether

318 the model could uniquely identify clusters of eggs that belonged together. The entire process

319 (creating a training set, training the random forest model and testing the model) was repeated

3201000 times.

322 For the unlabelled dataset, we calculated how many times in each of these 1000 runs the target 323 egg was matched with a cluster of eggs that were from the same female. If the percentage was 324 greater than 95\%, we considered this egg as a candidate for being from this female. To 325 corroborate this conclusion, we used non-phenotypic data e.g. laying dates, laying locality and 326 host species.

Phenotypic-genotypic similarity

329 We had genetic samples for 9 of the adult females, allowing us to create a genetic distance matrix.

330 To compare the phenotypic-genotypic similarity between these females and their eggs, we 331 created a trait distance matrix by taking means of the phenotypic parameters from their egg data, 332 and then using Euclidean distance as the distance metric. We compared the genetic distance 333 matrix with the trait distance matrix using a Mantel test, a statistical test of the correlation between 334 two matrices, implemented in the vegan package in $\mathrm{R}$ using the Kendall method (as this is most 335 appropriate for a small dataset). We also split the phenotype data into different components 
336 (spectral, pattern and shape) and calculated the phenotype-genotype similarities for each of these

337 components separately, to test whether different aspects of the egg phenotype are differentially

338 correlated with the female genotypes.

340 Results

341 Within- and between-female variability in egg appearance

342 Within-female variance was variable, with some females having very similar eggs (e.g. female 13

$343-$ within-female variance $=0.33$, Fig. 2 in Supplementary material) and others having relatively

344 high variance (e.g. female 29 - within-female variance =1.31, Fig. 4 in Supplementary material).

345 The between-female variance (comparing across females, using an "average egg" for each

346 female) was 1.832 (mean of trait standard deviations, $n=11$ traits; $S D=1.021$ ). Overall, within-

347 female variance $($ mean $=0.850, S D=0.295)$ was smaller than between-female variance (one

348 sample t test, $\mathrm{t}=14.87 \mathrm{df}=19, \mathrm{p}<0.001)$. Variability in the egg appearance is also visible in Fig.

3491 where the two most informative variables in the random forest analysis (PC2 for pattern and

350 PC2 for spectral data), are plotted (see below and Table 2). 


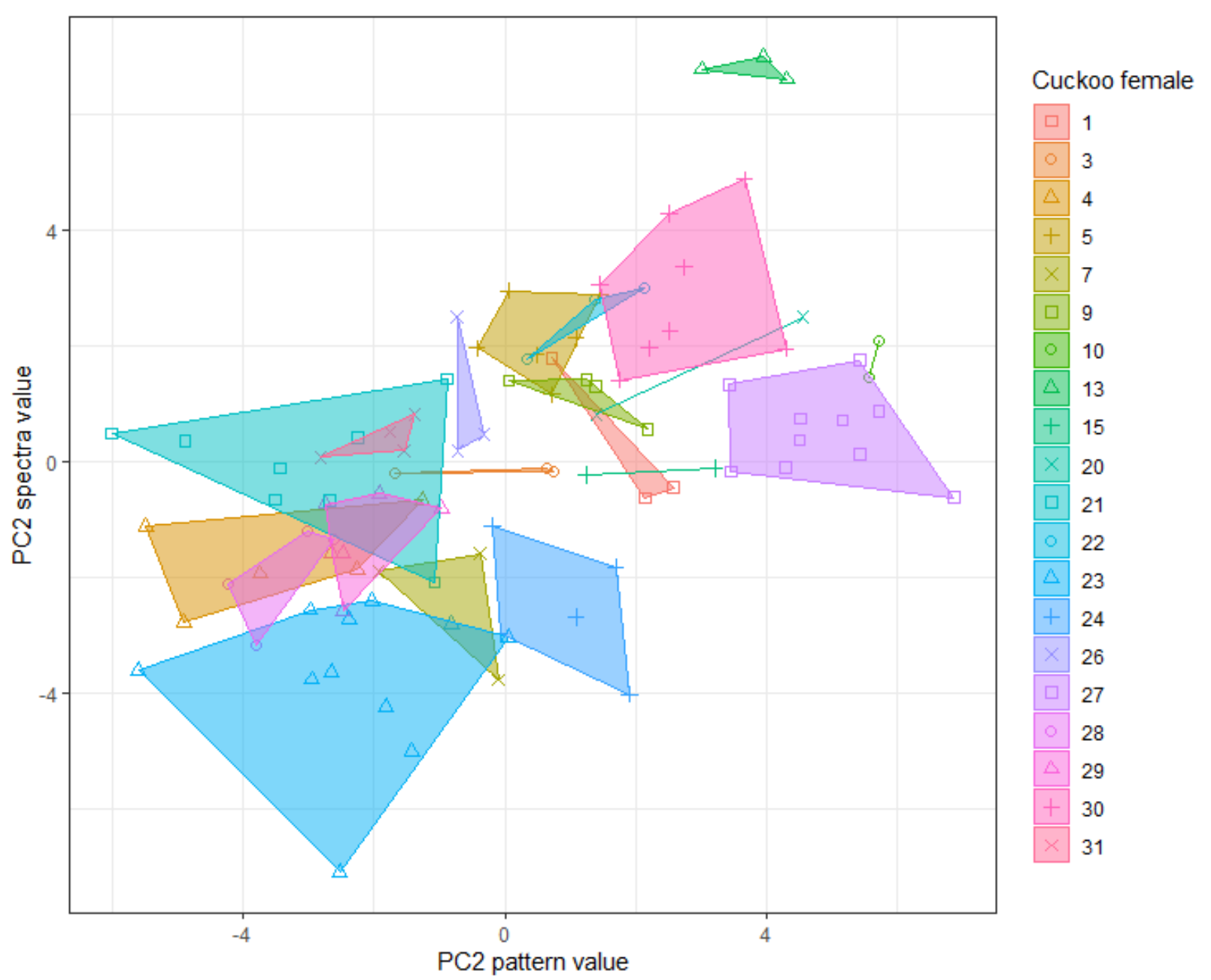

363 Figure 1 Values for individual eggs on the two most important $P C$ variables (according to the 364 random forest model), grouped by cuckoo female. PCA2 pattern variable indicates egg skew and 365 PC2 spectra variable indicates blueness/greenness of eggs (for details, see Table 2).

Human assessment

369 Participants with some experience of working with biological data performed better at the grouping 370 task than those with no experience, though there is no clear evidence that specific experience of 371 working with cuckoo eggs is beneficial (Table 1). 
Table 1. Cluster similarities of egg sorting performed by humans both without knowledge (when

373 they did not know the number of females) and with a known number of females.

\begin{tabular}{|l|l|l|}
\hline Group & No knowledge & Known number of females \\
\hline No experience $(\mathrm{n}=5)$ & $0.225(0.066)$ & $0.241(0.041)$ \\
\hline Non-specific experience $(\mathrm{n}=3)$ & $0.502(0.170)$ & $0.496(0.057)$ \\
\hline Specific experience $(\mathrm{n}=4)$ & $0.417(0.050)$ & $0.456(0.158)$ \\
\hline
\end{tabular}

Unsupervised learning

377 Clustering using unsupervised hierarchical learning gave a cluster similarity value of 0.452; similar

378 to that of experienced human observers, but better than inexperienced observers.

\section{Supervised learning (random forest analysis)}

$381 \quad$ Female clustering

382 Clustering using supervised random forest analysis (with a leave-one-out protocol) led to good

383 classification, with a mean of $77.08 / 95$ (81.1\%) of eggs correctly assigned to their genetic parent.

384 The cluster similarity had a mean of $0.61(S D=0.03)$, higher than both experienced human assessment and unsupervised learning.

387 We assessed variable importance (Table 2) using a full model including all data. PC2 for pattern was the most important variable for classification, and the variables loading onto this PC were predominantly those for the 'skew' of the pattern. PC2 for spectra was also important, with this variable being influenced by the 'blueness/greenness' of the egg. Finally, PC3 and PC1 for shape

391 were also informative. The variables loading onto these PCs were the length, width, volume and 392 surface area of the egg. 
394 Table 2 The importance of individual variables in egg clustering using random forest analysis.

\begin{tabular}{|c|c|c|}
\hline Variable & Mean decrease in accuracy & Main PCA loadings \\
\hline PC2_pattern & 28.42 & $\begin{array}{l}\text { Skew values at pattern } \\
\text { energy scales } 1,0.707,0.5 \text {, } \\
0.3536,0.25,0.1768,0.125 \text {, } \\
0.08839,0.0625,0.04419\end{array}$ \\
\hline PC2_spectra & 26.8 & $\begin{array}{l}426,447,468,489,510 \\
531 \mathrm{~nm}\end{array}$ \\
\hline PC3_shape & 23.81 & Length, max width \\
\hline PC1_shape & 21.37 & $\begin{array}{l}\text { Length, max width, volume, } \\
\text { surface area }\end{array}$ \\
\hline PC1_spectra & 19.79 & $\begin{array}{l}342,552,573,594,636,678 \\
699 \mathrm{~nm}\end{array}$ \\
\hline UV_shape & 19.36 & - \\
\hline PC2_shape & 16.91 & Ellipse deviation \\
\hline PC1_luminance & 15.42 & $\begin{array}{l}\text { Luminance sections } 1,2 \text { and } \\
3 \text {, standard deviation sections } \\
1,2 \text { and } 3\end{array}$ \\
\hline PC3_pattern & 15.18 & $\begin{array}{l}\text { Pattern energy scales } 1 \text {, } \\
0.7071,0.5,0.3536,0.04419 \text {, } \\
0.03125,0.0221\end{array}$ \\
\hline Brightness & 12.9 & - \\
\hline PC1_pattern & 11.23 & $\begin{array}{l}\text { Pattern energy scales } 0.3536 \text {, } \\
0.25,0.1768,0.125,0.08839 \text {, } \\
0.0625 \text {, total pattern energy, } \\
\text { total pattern energy in } \\
\text { segment } 2\end{array}$ \\
\hline
\end{tabular}

Variables with larger mean decrease in accuracy are more important for classifying the data

396 (mean decrease in accuracy is a measure of how much the accuracy of the random forest

397 decreases due to the exclusion/permutation of a single variable). The main PCA loadings are

398 those that were greater than $+/-0.25$. 
Same/different analysis

402 For our labelled data, on average, $77.46 / 95$ (81.5\%) of eggs were matched with themselves.

403 During the training phase, the model was always trained with 'same' pairs that consisted of two

404 different eggs from the same female, so this was done as a test of whether the model was able

405 to recognise two truly identical eggs as coming from the same female.

406 In addition, on average 73.50/95 (77.4\%) eggs were uniquely matched with other eggs laid by the

407 same female.

408 Out of 87 unlabelled eggs, the model was able to reliably (on $95 \%$ of runs) identify 22 as belonging

409 to a labelled female (9 eggs assigned to female 5, 5 eggs to female 27, 3 eggs to female 13 , and

4101 egg to each of females $4,21,28,29,30)$.

Phenotypic-genotypic similarity

413 There was no significant relationship between overall female egg phenotype distance and female 414 genetic distance (Mantel test $r=0.1968, p=0.098$, Fig. 2).

A

(30)

416

30)

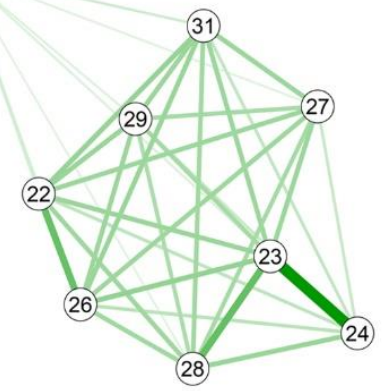

B

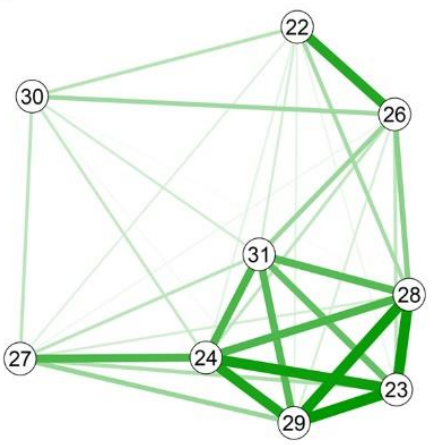

C

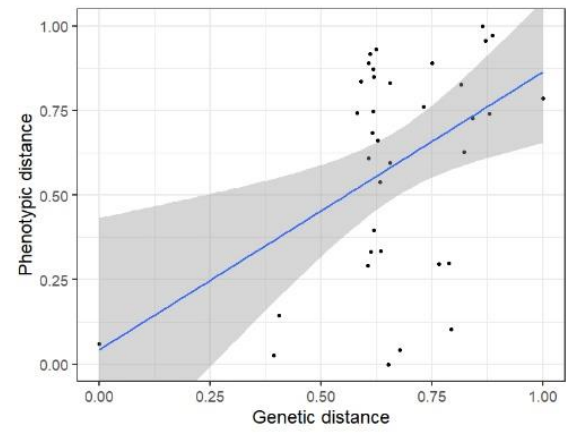

417 Figure 2 Phenotypic distances of nine average eggs laid by nine genotyped common cuckoo

418 females (A) and their genetic distances (B). Thicker green lines denote higher phenotypic and 419 genetic similarity. Correlation between phenotypic and genetic distances (C). 
421 When considering each aspect of phenotypic similarity separately, both pattern/luminance and 422 shape distance metrics did not correlate with genotypic similarity $(r=0.0254, p=0.3861$ and $r=$ $423-0.2317, p=0.9256$ respectively, Fig. 3A-D). However, spectral similarity did correlate with genetic 424 similarity $(r=0.356, p=0.037$, Fig. 3E-F $)$.

A

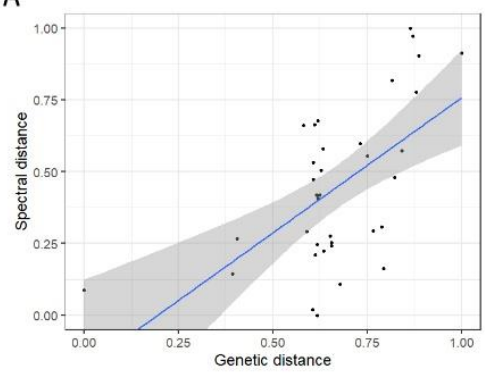

D

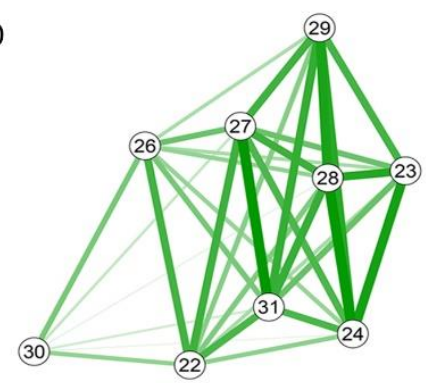

B

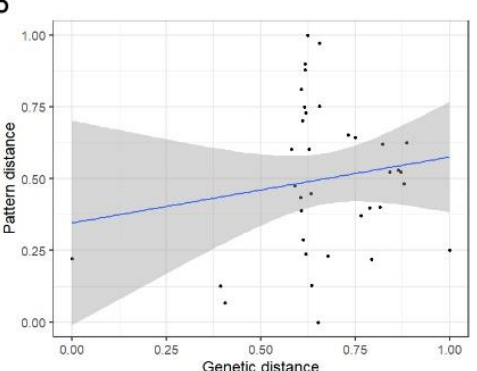

E

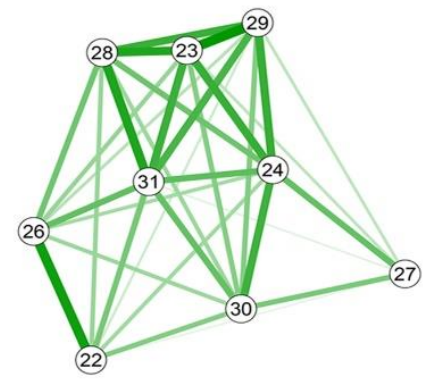

C

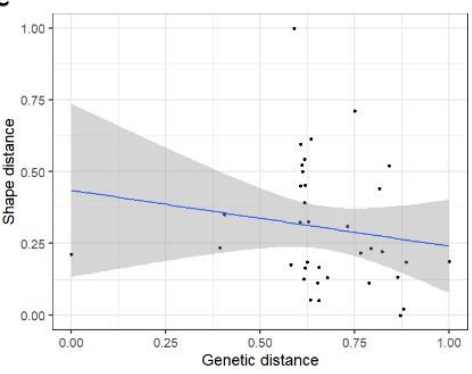

$\mathrm{F}$

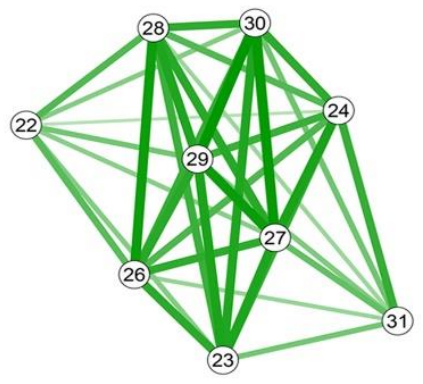

Figure 3 Correlation between spectral (A), pattern/lumiance (B) and shape (C) distances, respectively and genetic distances. Individual phenotypic distances of average eggs laid by nine genotyped common cuckoo females: spectral $(D)$, pattern/luminance $(E)$ and shape $(F)$ distances.

Discussion

433 The results of our study support the 'constant egg-type hypothesis' predicting that individual 434 cuckoo females lay eggs with a relatively constant appearance (Latter 1902, Chance 1940, Baker 435 1942, Lack 1968, Wyllie 1981, Moksnes et al. 2008). We confirmed that the within-clutch variation of cuckoo egg appearance is significantly lower than between-clutch variation. This has also been 
437 observed in other bird species and several adaptive explanations have been proposed for this

438 phenomenon, such as easier recognition of the parasitic egg by hosts (Øien et al., 1995),

439 recognition of an individual's own clutch in colonially-breeding birds (Hauber et al., 2019) or

440 signalling the female quality (Moreno \& Osorno, 2003). Moreover, it has been shown that diet has

441 only a small effect on eggshell colour and that this trait is more affected by maternal identity,

442 suggesting that egg colour may carry information about intrinsic properties of the female

443 (Dearborn, Hanley, Ballantine, Cullum, \& Reeder, 2012). Therefore, it seems that there is the

444 potential to use egg appearance to identify individual bird females.

446 Here, we demonstrated that the unsupervised computer-vision based classifier can outperform

447 human participants (especially inexperienced ones) in assigning cuckoo eggs to correct female

448 clusters. Human egg classifiers with experience of handling natural eggs (either cuckoo or of other

449 species) were able to more accurately sort them compared to people without this experience

450 (Table 1). This may indicate that experienced observers have some knowledge of which cues are

451 likely to be useful. They may also be more likely to be practiced at this type of fine discrimination

452 task, or be more motivated to take part, given their interest in biology. Interestingly, knowing the

453 number of groups (here: cuckoo females that laid eggs) did not increase sorting accuracy

454 substantially.

456 The automatic hierarchical clustering method showed very similar results to experienced human 457 classifiers, while supervised random forest analysis showed considerably better results and $81 \%$ 458 of cuckoo eggs were assigned correctly. This suggests that in some cases, automatic assessment 459 methods may be preferable to human assessment. Detailed consideration of the clusters created 460 by humans and the automatic methods showed that the same females were problematic for both 461 clustering methods, probably reflecting phenotypic overlap between some individuals (all sorting 462 results can be found in Supplementary Material). Our results showed that one of the pattern 
characteristics (skew), blueness of colour and finally egg size were the most important parameters

464 that helped the automatic method to cluster eggs the most accurately. The slight improvement in

465 clustering accuracy for the automatic methods over human assessment may reflect the use of

466 features that humans are not able to see (e.g. the UV peak of the spectra). Therefore, we would

467 recommend automatic assessment over human assessment where possible.

469 A previous study suggested that closely related cuckoo females may lay eggs that are 470 indistinguishable from each other (Moksnes et al., 2008). Our limited dataset of nine caught 471 females for which we calculated genetic distances partially supports this idea as we found that 472 the background colour of eggs was more similar between closely related females. However, 473 pattern and dimension distances between females did not correlate with their genetic distances. 474 As an interesting example, we caught two closely related females, mother (female 23) and 475 daughter (female 24), and we photographed and measured the colour of their eggs. From the 476 photographs, we can see that their eggs look very similar in the colour and spotting pattern; 477 however, they differ considerably in size (Fig 3 in Supplementary material). Similarly, we can also 478 see the resemblance in egg colour and pattern (but not in size) between other closely related 479 females (females 23 vs 28 and females 22 vs 26; Fig. 3 and 4 in Supplementary material). 480 Previous studies showed that all investigated egg features - colour, spotting pattern and also size 481 have high heritability (Wei et al., 1992; Collias, 1993; Gosler et al., 2000; Christians, 2002; 482 Morales et al., 2010). Our results indicate that the background colour might be a more heritable 483 trait than spotting pattern and egg size or shape, which supports the idea that egg colour is of 484 vital importance for egg recognition (Avilés et al., 2010; Spottiswoode \& Stevens, 2010; Michal 485 Šulc, Procházka, Capek, \& Honza, 2016). Since several studies reported that hosts and even 486 parasites themselves also use spotting pattern (López-de-Hierro \& Moreno-Rueda, 2010; 487 Spottiswoode \& Stevens, 2010; de la Colina, Pompilio, Hauber, Reboreda, \& Mahler, 2012) or 488 egg size (Marchetti, 2000; Spottiswoode, 2013) when recognizing and eliminating parasitic eggs, 
489 we still expect relatively high heritability of these egg traits in brood parasites. We suspect that

490 the insignificant relationship between genetic distance and phenotypic distance in spotting pattern

491 and size is only a matter of our limited sample size. A Larger sample size, including more mother-

492 daughter pairs, is needed to truly estimate heritability values of individual egg traits (de

493 Villemereuil, Gimenez, \& Doligez, 2013). The lack of significant correlation between egg shape

494 and genetic similarity may also be explained by the fact that egg size often reflects the size of

495 laying females (Larsson \& Forslund, 1992; Nager \& Zandt, 1994), which depends on the genetic

496 contribution of both parents and therefore might differ more even in closely related females.

497 Moreover, even within one host population, cuckoos are raised by host parents that vary in their

498 provisioning care (Požgayová, Piálková, Honza, \& Procházka, 2018), which may also influence

499 the body size of cuckoo females in adulthood. Finally, some studies showed there is a positive

500 relationship between food availability and egg size (reviewed in Christians 2002). Consequently,

501 since egg size and shape may even differ in such closely related females, these traits may be

502 used for the separation of their eggs. And indeed, this is what we observed in several of our

503 human participants and also in computer analysis where separation of mother and daughter eggs

504 was relatively straightforward presumably because of the apparent difference in the size and

505 shape (see Supplementary material).

507 Although our results are in concordance with a previous study showing that the visual appearance

508 of cuckoo eggs cannot be used to assign them reliably to individual females without genetic data

509 (Moksnes et al. 2008), here we present a new approach that uses both genetic and phenotypic

510 information. We used this method for assigning cuckoo eggs for which we did not have genetic

511 data (because they were ejected by hosts or predated), allowing us to assign 22 eggs (out of 87)

512 to eight known females. This method seemed to work well for females that laid very distinctive

513 eggs and therefore results will strongly depend on within- and between-clutch variation. We may

514 expect better results of the method in species where between-clutch variation substantially 
515 exceeds the within-clutch variation. It must also be noted that the accuracy of the assignment will

516 increase with the relative number of (genetically and phenotypically) analysed samples in the

517 study area that are used for the training dataset, because broad sampling will reduce the chance

518 that an unsampled egg that has been laid by a completely new female will be assigned to an

519 existing (incorrect) female. Finally, it is important to apply other information (laying date and laying

520 area) to eliminate potential incorrect assignments. However, in our dataset, we did not find any

521 such discrepancies for the 22 eggs that were automatically assigned based on their phenotypes.

523 We conclude that egg appearance alone cannot be used to identify individual cuckoo females.

524 Clusters created either by people using egg photographs or by the computer using spectral and

525 image data did not fully correspond to the true female identity acquired from molecular analyses,

526 though the supervised automatic assessment was the most accurate for classification. Our results

527 support the idea that more closely related females lay eggs more similar in their colour. However,

528 the size and shape of the eggs seems to be the least heritable trait, which may substantially help

529 to distinguish even between eggs of closely related cuckoo females. We advocate genetic

530 analysis to be used for determination of maternity in this species. However, in sufficiently sampled

531 systems, supervised analytical methods that use egg visual features might additionally help to

532 broaden sample sizes, which may be very desirable for studying biological questions (see e.g.

533 Koleček et al. 2020 in prep). We encourage researchers investigating inter- and intra-specific

534 brood parasitism to use this low-cost and ethically more appropriate method of individual

535 identification. Since a similar technique has been successfully used in non-parasitic species

536 (Höltje et al., 2016), identification of laying females using egg appearance therefore has the

537 potential to be of widespread use.

538

539 


\section{Acknowledgements}

542 We would like to thank Lisandrina Mari, Kamila Bendová, Kristýna Míčková, Jana Hodanová,

543 Klára Stehlíková, Martina Fridrichová, Marta Potůčková, David Tesař, Václav Jelínek and Petr

544 Potůček for their sorting of cuckoo eggs. Lisandrina Mari also substantially helped with writing of

545 the genetic part of methods. We also thank Lukáš Kulísek, Boris Prudík, Kateřina Sosnovcová,

546 Jaroslav Koleček and Miroslav Čapek for help with fieldwork. We thank Micha Elsner for his help

547 with data analyses. We are also very grateful to Vladimír Beneš and the European Molecular

548 Biology Laboratory Genomic Core Facility in Heidelberg (Germany) for their kind advice and

549 technical support regarding Illumina sequencing. The managers of the Hodonín Fish Farm kindly

550 permitted us conducting the fieldwork on their grounds.

\section{Ethical note}

553 This study was carried out with the permission of the regional nature conservation authorities

554 (permit numbers JMK: $115874 / 2013$ and 38506/2016; MUHOCJ: 41433/2012/OŽP, $55534437 / 2014 / \mathrm{OŽP}$, and 14306/2016/OŽP). The fieldwork adhered to the animal care protocol 556 (experimental project numbers 039/2011 AV ČR and 3030/ENV/17-169/630/17) and to the Czech

557 Law on the Protection of Animals against Mistreatment (license numbers CZ 01272 and CZ 558 01284). This study was carried out with the permission of the regional nature conservation 559 authorities (permit numbers JMK: 115874/2013 and 38506/2016; MUHOCJ: 41433/2012/OŽP, 560 34437/2014/OŽP, and 14306/2016/OŽP).

\section{Funding}

563 This work was supported by the Czech Science Foundation (grant number 17-12262S) and by 564 the Programme for research and mobility of young researchers of the Czech Academy of 565 Sciences (MSM200931801, awarded to M.Š.). 


\section{References}

568 Ådahl, E., Lindström, J., Ruxton, G. D., Arnold, E. K., \& Begg, T. (2004). Can intraspecific brood parasitism be detected using egg morphology only? Journal of Avian Biology, 35(4), 360-364. doi:10.1111/j.0908-8857.2004.03224.x

Avilés, J. M., Vikan, J. R., Fossøy, F., Antonov, A., Moksnes, A., Røskaft, E., \& Stokke, B. G. (2010). Avian colour perception predicts behavioural responses to experimental brood parasitism in chaffinches. Journal of Evolutionary Biology, 23(2), 293-301. doi:10.1111/j.1420-9101.2009.01898.x

Bensch, S. (1996). Female Mating Status and Reproductive Success in the Great Reed Warbler: Is there a Potential Cost of Polygyny that Requires Compensation. Journal of

Berg, C. P. van den, Troscianko, J., Endler, J. A., Marshall, N. J., \& Cheney, K. L. (2020). analysis of colour patterns in nature. Methods in Ecology and Evolution, 11(2), 316-332.

Blumstein, D. T., Mennill, D. J., Clemins, P., Girod, L., Yao, K., Patricelli, G., ... Kirschel, A. N. G. (2011). Acoustic monitoring in terrestrial environments using microphone arrays:

Brown, C. R., \& Sherman, L. C. (1989). Variation in the appearance of swallow eggs and the applications, technological considerations and prospectus. Journal of Applied Ecology, 48(3), 758-767. doi:10.1111/j.1365-2664.2011.01993.x

Bolger, D. T., Morrison, T. A., Vance, B., Lee, D., \& Farid, H. (2012). A computer-assisted system for photographic mark-recapture analysis. Methods in Ecology and Evolution, detection of intraspecific brood parasitism. The Condor, 91(3), 620-627. unreliable in determining maternity in communal clutches of guira cuckoos Guira guira. 
594 Chance, E. P. (1922). The cuckoo's secret (pp. 1-290). London : Sidgwick \& Jackson,. doi:10.5962/bhl.title.161352

Christians, J. K. (2002). Avian egg size: variation within species and inflexibility within individuals. Biological Reviews, 77(1), 1-26. doi:https://doi.org/10.1017/S1464793101005784

Christin, S., Hervet, É., \& Lecomte, N. (2019). Applications for deep learning in ecology. Methods in Ecology and Evolution, 10(10), 1632-1644. doi:10.1111/2041-210X.13256

Collias, E. C. (1993). Inheritance of egg-color polymorphism in the village weaver (Ploceus cucullatus). The Auk, 110(4), 683-692.

Crall, J. P., Stewart, C. V., Berger-Wolf, T. Y., Rubenstein, D. I., \& Sundaresan, S. R. (2013). HotSpotter - Patterned species instance recognition. In 2013 IEEE Workshop on Applications of Computer Vision (WACV) (pp. 230-237). doi:10.1109/WACV.2013.6475023 recognition cues reveal the decision rules used for egg rejection by hosts of a variably de Villemereuil, P., Gimenez, O., \& Doligez, B. (2013). Comparing parent-offspring regression mimetic avian brood parasite. Animal Cognition, 15(5), 881-889.

615 Dearborn, D. C., Hanley, D., Ballantine, K., Cullum, J., \& Reeder, D. M. (2012). Eggshell colour with frequentist and Bayesian animal models to estimate heritability in wild populations: a simulation study for Gaussian and binary traits. Methods in Ecology and Evolution, $4(3), 260-275$. is more strongly affected by maternal identity than by dietary antioxidants in a captive poultry system. Functional Ecology, 26(4), 912-920. doi:https://doi.org/10.1111/j.13652435.2012.02001.x 
Deb, D., Wiper, S., Gong, S., Shi, Y., Tymoszek, C., Fletcher, A., \& Jain, A. K. (2018). Face Recognition: Primates in the Wild. In 2018 IEEE 9th International Conference on Biometrics Theory, Applications and Systems (BTAS) (pp. 1-10). doi:10.1109/BTAS.2018.8698538

Eadie, J. M., Smith, J. N. M., Zadworny, D., Kühnlein, U., \& Cheng, K. (2010). Probing parentage in parasitic birds: an evaluation of methods to detect conspecific brood parasitism using goldeneyes Bucephala islandica and BI. clangula as a test case. Journal of Avian Biology, 41(2), 163-176. doi:10.1111/j.1600-048X.2009.04735.x

Ferreira, A. C., Silva, L. R., Renna, F., Brandl, H. B., Renoult, J. P., Farine, D. R., ... Doutrelant, C. (2020). Deep learning-based methods for individual recognition in small birds.

Fossøy, F., Antonov, A., Moksnes, A., Røskaft, E., Vikan, J. R., Møller, A. P., ... Stokke, B. G. (2011). Genetic differentiation among sympatric cuckoo host races: males matter. Proceedings of the Royal Society B: Biological Sciences, 278(1712), 1639-1645. doi:10.1098/rspb.2010.2090

634 Fossøy, F., Moksnes, A., Røskaft, E., Antonov, A., Dyrcz, A., Moskat, C., ... Stokke, B. G. (2012). Sex Allocation in Relation to Host Races in the Brood-Parasitic Common Cuckoo (Cuculus canorus). PLOS ONE, 7(5), e36884. doi:10.1371/journal.pone.0036884

Gibbons, D. W. (1986). Brood parasitism and cooperative nesting in the moorhen, Gallinula chloropus. Behavioral Ecology and Sociobiology, 19(3), 221-232.

642 Gloag, R., Fiorini, V. D., Reboreda, J. C., \& Kacelnik, A. (2013). The wages of violence: (2000). Genetic evidence for female host-specific races of the common cuckoo. Nature, mobbing by mockingbirds as a frontline defence against brood-parasitic cowbirds. 
645 Gosler, A. G., Barnett, P. R., \& James Reynolds, S. (2000). Inheritance and variation in eggshell patterning in the great tit Parus major. Proceedings of the Royal Society of London. Series B: Biological Sciences, 267(1461), 2469-2473.

Griffith, S. C., Barr, I., Sheldon, B. C., Rowe, L. V., \& Burke, T. (2009). Egg patterning is not a

GrønstøI, G., Blomqvist, D., \& Wagner, R. H. (2006). The Importance of Genetic Evidence for Identifying Intra-Specific Brood Parasitism. Journal of Avian Biology, 37(2), 197-199.

Hanley, D., Šulc, M., Brennan, P. L., Hauber, M. E., Grim, T., \& Honza, M. (2016). Dynamic egg color mimicry. Ecology and Evolution, 6(12), 4192-4202.

Hansen, M. F., Smith, M. L., Smith, L. N., Salter, M. G., Baxter, E. M., Farish, M., \& Grieve, B. (2018). Towards on-farm pig face recognition using convolutional neural networks. Computers in Industry, 98, 145-152. doi:10.1016/j.compind.2018.02.016

Hauber, M. E., Luro, A., McCarty, C. J., Barateli, K., Cassey, P., Hansen, E. S., \& Dale, J. (2019). Interannual repeatability of eggshell phenotype in individual female Common Murres ( Uria aalge ). Canadian Journal of Zoology, 97(4), 385-391. doi:10.1139/cjz-

Hiby, L., Lovell, P., Patil, N., Kumar, N. S., Gopalaswamy, A. M., \& Karanth, K. U. (2009). A tiger cannot change its stripes: using a three-dimensional model to match images of living tigers and tiger skins. Biology Letters, 5(3), 383-386. doi:10.1098/rsbl.2009.0028

666 Höltje, H., Mewes, W., Haase, M., \& Ornés, A. S. (2016). Genetic evidence of female specific eggshell colouration in the Common Crane (Grus grus). Journal of Ornithology, 157(2),

669 Honza, M., Vošlajerová, K., \& Moskát, C. (2007). Eviction behaviour of the common cuckoo Cuculus canorus chicks. Journal of Avian Biology, 38(3), 385-389. 
672 Hou, J., He, Y., Yang, H., Connor, T., Gao, J., Wang, Y., ... Zhou, S. (2020). Identification of animal individuals using deep learning: A case study of giant panda. Biological Conservation, 242, 108414. doi:10.1016/j.biocon.2020.108414

Jackson, W. M. (1992). Estimating Conspecific Nest Parasitism in the Northern Masked Weaver

Jelínek, V., Šulc, M., Štetková, G., \& Honza, M. (2020). Fast and furious: host aggression modulates behaviour of brood parasites. BioRxiv, 2020.04.27.063933. doi:10.1101/2020.04.27.063933

Jones, O. R., \& Wang, J. (2010). COLONY: a program for parentage and sibship inference from multilocus genotype data. Molecular Ecology Resources, 10(3), 551-555. doi:10.1111/j.1755-0998.2009.02787.x

Jung, T. S., Boonstra, R., \& Krebs, C. J. (2020). Mark my words: experts' choice of marking methods used in capture-mark-recapture studies of small mammals. Journal of Mammalogy, 101(1), 307-317.

Kearse, M., Moir, R., Wilson, A., Stones-Havas, S., Cheung, M., Sturrock, S., ... Drummond, A. (2012). Geneious Basic: an integrated and extendable desktop software platform for the organization and analysis of sequence data. Bioinformatics (Oxford, England), 28(12),

691 Krause, J., Krause, S., Arlinghaus, R., Psorakis, I., Roberts, S., \& Rutz, C. (2013). Reality mining of animal social systems. Trends in Ecology \& Evolution, 28(9), 541-551.

694 Langmead, B., \& Salzberg, S. L. (2012). Fast gapped-read alignment with Bowtie 2. Nature Methods, 9(4), 357-359. doi:10.1038/nmeth.1923

696 Larsson, K., \& Forslund, P. (1992). Genetic and social inheritance of body and egg size in the 
barnacle goose (Branta leucopsis). Evolution, 46(1), 235-244.

698 Lemons, P. R., Sedinger, J. S., \& Randle, P. S. (2011). Detecting conspecific brood parasitism using egg morphology in black brant Branta bernicla nigricans. Journal of Avian Biology, 42(4), 282-288. doi:10.1111/j.1600-048X.2011.05217.x

702

Lindberg, M. S. (2012). A review of designs for capture-mark-recapture studies in discrete time. Journal of Ornithology, 152(2), 355-370.

López-de-Hierro, M. D. G., \& Moreno-Rueda, G. (2010). Egg-spot pattern rather than egg colour affects conspecific egg rejection in the house sparrow (Passer domesticus). Behavioral Ecology and Sociobiology, 64(3), 317-324. doi:10.1007/s00265-009-0811-9 parasitism. Nature, 422(6931), 495-499.

Lyon, Bruce E. (1993). Tactics of parasitic American coots: host choice and the pattern of egg dispersion among host nests. Behavioral Ecology and Sociobiology, 33(2), 87-100. perspective. Annual Review of Ecology, Evolution, and Systematics, 39, 343-363. spatial analysis of colour in r. Methods in Ecology and Evolution, 10(7), 1097-1107. doi:10.1111/2041-210X.13174

Marchetti, K. (2000). Egg rejection in a passerine bird: size does matter. Animal Behaviour, 59(4), 877-883.

McMaster, D. G., Neudorf, D. L. H., Sealy, S. G., \& Pitcher, T. E. (2004). A comparative analysis parasite-host relationships determined by DNA fingerprinting. Behavioral Ecology and Sociobiology, 38(2), 115-129. doi:10.1007/s002650050224 
723 Moksnes, A., Røskaft, E., Rudolfsen, G., Skjelseth, S., Stokke, B. G., Kleven, O., ... Taborsky, M. (2008). Individual female common cuckoos Cuculus canorus lay constant egg types but egg appearance cannot be used to assign eggs to females. Journal of Avian Biology, 39(2), 238-241. doi:10.1111/j.2008.0908-8857.04158.x

728

Møller, A. P. (1987). Intraspecific nest parasitism and anti-parasite behaviour in swallows,

729 Hirundo rustica. Animal Behaviour, 35(1), 247-254. doi:10.1016/S0003-3472(87)80230-

Morales, J., Kim, S.-Y., Lobato, E., Merino, S., Tomás, G., MARTíNEZ-de la PUENTE, J., \& Moreno, J. (2010). On the heritability of blue-green eggshell coloration. Journal of Evolutionary Biology, 23(8), 1783-1791.

Moreno, J., \& Osorno, J. L. (2003). Avian egg colour and sexual selection: does eggshell pigmentation reflect female condition and genetic quality? Ecology Letters, 6(9), 803806.

Nager, R. G., \& Zandt, H. S. (1994). Variation in egg size in great tits. ARDEA-WAGENINGEN-, 82, 315-315.

Øien, I. J., Moksnes, A., \& Røskaft, E. (1995). Evolution of variation in egg color and marking pattern in European passerines: adaptations in a coevolutionary arms race with the cuckoo, Cuculus canorus. Behavioral Ecology, 6(2), 166-174. doi:10.1093/beheco/6.2.166

Paillisson, J.-M., Latraube, F., Marion, L., \& Bretagnolle, V. (2008). Indirect evidence of conspecific nest parasitism in the colonial whiskered tern (Chlidonias hybrida). Comptes Rendus Biologies, 331(7), 559-567. doi:10.1016/j.crvi.2008.04.010

Petersen, M. R. (1992). Intraspecific Variation in Egg Shape among Individual Emperor Geese Payne, R. B. (1973). Individual Laying Histories and the Clutch Size and Numbers of Eggs of Parasitic Cuckoos. The Condor, 75(4), 414-438. doi:10.2307/1366563 
Petrusková, T., Pišvejcová, I., Kinštová, A., Brinke, T., \& Petrusek, A. (2016). Repertoire-based individual acoustic monitoring of a migratory passerine bird with complex song as an

Petrželková, A., Pöysä, H., Klvaňa, P., Albrecht, T., \& Hořák, D. (2017). Egg morphology fails to identify nests parasitized by conspecifics in common pochard: a test based on protein fingerprinting and including female relatedness. Journal of Avian Biology, 48(2), 229_

Piálek, L., Burress, E., Dragová, K., Almirón, A., Casciotta, J., \& Říčan, O. (2019). Phylogenomics of pike cichlids (Cichlidae: Crenicichla) of the C. mandelburgeri species complex: rapid ecological speciation in the Iguazú River and high endemism in the conspecific brood parasitism in goldeneyes Bucephala clangula examined using protein fingerprinting. Journal of Avian Biology, 40(4), 453-456. doi:10.1111/j.1600-

Požgayová, M., Piálková, R., Honza, M., \& Procházka, P. (2018). Sex-specific nestling growth in an obligate brood parasite: Common Cuckoo males grow larger than females. The Auk: Ornithological Advances, 135(4), 1033-1042.

Ptacek, L., Machlica, L., Linhart, P., Jaska, P., \& Muller, L. (2016). Automatic recognition of bird

772 Purcell, S., Neale, B., Todd-Brown, K., Thomas, L., Ferreira, M. A. R., Bender, D., ... Sham, P. individuals on an open set using as-is recordings. Bioacoustics, 25(1), 55-73. 
775 Ramey, J. A. (2012). clusteval: Evaluation of Clustering Algorithms. Retrieved from

776 https://CRAN.R-project.org/package=clusteval

777 Roy, C. L., Parker, P. G., \& Gates, R. J. (2009). Egg Morphology is an Unreliable Indicator of

778 Intraspecific Nest Parasitism in Wood Ducks. The Condor, 111(2), 377-381.

779 doi:10.1525/cond.2009.080117

780 Soler, M. (Ed.). (2017). Avian Brood Parasitism. Cham, Switzerland: Springer International

$781 \quad$ Publishing.

782 Spottiswoode, C. N. (2013). A brood parasite selects for its own egg traits. Biology Letters, 9(5),

$783 \quad 20130573$.

784 Spottiswoode, C. N., \& Stevens, M. (2010). Visual modeling shows that avian host parents use

785 multiple visual cues in rejecting parasitic eggs. Proceedings of the National Academy of

$786 \quad$ Sciences, 107(19), 8672-8676.

787 Stone, M. (1974). Cross-validatory choice and assessment of statistical predictions. Journal of the Royal Statistical Society: Series B (Methodological), 36(2), 111-133.

789 Šulc, M., Štětková, G., Jelínek, V., Czyż, B., Dyrcz, A., Karpińska, O., ... Honza, M. (2020).

790 Killing behaviour of adult brood parasites. Behaviour, 157(12-13), 1099-1111.

$791 \quad$ doi:10.1163/1568539X-bja10033

792 Šulc, Michal, Procházka, P., Capek, M., \& Honza, M. (2016). Birds use eggshell UV reflectance when recognizing non-mimetic parasitic eggs. Behavioral Ecology, 27(2), 677-684.

794 Šulc, Michal, Troscianko, J., Štětková, G., Hughes, A. E., Jelínek, V., Capek, M., \& Honza, M. (2019). Mimicry cannot explain rejection type in a host-brood parasite system. Animal

797 Troscianko, J. (2014). A simple tool for calculating egg shape, volume and surface area from 798 digital images. Ibis, 156(4), 874-878.

799 Troscianko, J., \& Stevens, M. (2015). Image calibration and analysis toolbox-a free software 800 suite for objectively measuring reflectance, colour and pattern. Methods in Ecology and 
bioRxiv preprint doi: https://doi.org/10.1101/2020.11.25.398131; this version posted November 26,2020 . The copyright holder for this preprint

(which was not certified by peer review) is the author/funder, who has granted bioRxiv a license to display the preprint in perpetuity. It is made available under aCC-BY-NC-ND 4.0 International license.

Evolution, 6(11), 1320-1331.

802 Wei, R., Bitgood, J. J., \& Dentine, M. R. (1992). Inheritance of Tinted Eggshell Colors in White-

803 Shell Stocks1. Poultry Science, 71(3), 406-418. doi:10.3382/ps.0710406

804 Weinstein, B. G. (2018). A computer vision for animal ecology. Journal of Animal Ecology,

805 87(3), 533-545. doi:10.1111/1365-2656.12780

806 Wyllie, I. (1981). The Cuckoo. Universe Books.

807 